\title{
An online kernel-based clustering approach for value function approximation
}

\author{
N. Tziortziotis and K. Blekas \\ Department of Computer Science, University of Ioannina \\ P.O.Box 1186, Ioannina 45110 - Greece \\ \{ntziorzi,kblekas\}@cs.uoi.gr
}

\begin{abstract}
Value function approximation is a critical task in solving Markov decision processes and accurately representing reinforcement learning agents. A significant issue is how to construct efficient feature spaces from agent's samples in order to obtain optimal policy. This study addresses this challenge by proposing an online kernel-based clustering approach for building appropriate basis functions during the learning process. The method uses a kernel function capable of handling pairs of state-action as sequentially generated by the agent. At each time step, the procedure either adds new clusters, or adjusts the winning cluster's parameters. By considering the value function as a linear combination of the constructed basis functions, the weights are simultaneously optimized in a temporal-difference framework in order to minimize the Bellman approximation error. The proposed method is evaluated in numerous known simulated environments.
\end{abstract}

\section{Introduction}

The goal of Reinforcement Learning (RL) $[1,2]$ is to control an autonomous agent in unknown environments. The agent interacts with the environment which is typically modeled as a Markov Decision Process (MDP), and receives a scalar reward signal that evaluates the quality of the selected transitions. The decision making procedure is designed so as to choose actions with the optimum expected rewards. The quality of such a policy is quantified by the so-called value function which associates to every state the expected discounted reward when starting from this state and all decisions are made following this policy. However, in cases with large or infinite state spaces the value function cannot be calculated explicitly. In such domains a common strategy is to employ function approximation, by representing the value function as a linear combination of some set of basis functions.

The Temporal Difference (TD) family of algorithms [3] provide a nice framework for policy evaluation. The parameters of the value function are usually learned from data, as in the case of incremental TD and the Least-Squares TD (LSTD) methods $[4,5]$. Also, kernelized reinforcement learning methods have been paid a lot of attention by employing kernel techniques to standard RL methods [6] and Gaussian Processes for approximating the value function [7-9]. 
However, in most cases the basis functions used for estimating the value function remain fixed during the learning process, as for example a recent work presented in [10] uses fixed Fourier basis functions for value function approximation. Alternatively, A steady number of basis functions are tuned in a batch manner, as in the cases presented in $[11,12]$ that build a graph over the state space after selecting a large number of input data and then generates the $k$ eigenvectors of the graph Laplacian matrix. In another work in [13] a set of $k$ RBF basis function are adjusted directly over the Bellman's equation of the value function. Finally, in [14] the probability density function and the reward model, which are assumed to be known, are used for creating basis function from Krylov space vectors (powers of the transition matrix used to systems of linear equations).

In this paper we propose a novel framework for value function approximation which addresses the issue of online construction of basis functions. An online kernel-based clustering approach is used for separating the input space that contains pairs of state-action by appropriate considered a kernel function that encapsulates both kind of information. The clustering procedure selects iteratively a winning prototype and applies a learning procedure for the adaptation of its parameters based on stochastic gradient descent. In addition, it provides a mechanism for automatically adding clusters. The parameters of the clusters can be further used for building a dictionary of basis function which can be employed to the policy evaluation procedure for the adaptation of weights of the value function linear model. These two stages act simultaneously during the learning process aiming at estimating optimal policy after convergence. The proposed method has been tested to several known simulated environments where we have made comparisons with a recent value function approximation approach that uses fixed Fourier basis functions.

The remaining of this paper is organized as follows. In Section 2, we briefly we present some preliminaries and review the basic TD scheme for value function approximation. Section 3 contains the main contribution of this paper where we describe an efficient online kernel-based clustering algorithm for constructing basis functions and how it can be embedded to the basic TD learning scheme. In Section 4 we present experimental results and finally, in section 5 we give conclusions and suggestions for future research.

\section{Background and preliminaries}

A Markov Decision Process (MDP) is a tuple $(\mathcal{S}, \mathcal{A}, P, R, \gamma)$, where $\mathcal{S}$ is a set of states; $\mathcal{A}$ a set of actions; $P: \mathcal{S} \times \mathcal{A} \times S \rightarrow[0,1]$ is a Markovian transition model that specifies the probability $P\left(s, a, s^{\prime}\right)$ of transition to a state $\boldsymbol{s}^{\prime}$ when taken an action $a$ in state $s ; R: \mathcal{S} \rightarrow \mathbb{R}$ is the reward function for a stateaction pair; and $\gamma \in(0,1)$ is the discount factor for future rewards. A stationary policy $\pi: \mathcal{S} \rightarrow \mathcal{A}$ for a MDP is a mapping from states to actions and denotes a mechanism for choosing actions. An episode is a sequence of state transitions: 
$<s_{1}, a_{1}, r_{1}, s_{2}, \ldots,>$. An agent repeatedly chooses actions until the current episode terminates, and then a new episode starts over again.

The notion of value function is of central interest in reinforcement learning tasks. Given a policy $\pi$, the value $V^{\pi}(s)$ of a state $s$ is defined as the expected discounted sum of rewards obtained when starting from this state until the current episode terminates following policy $\pi$ :

$$
V^{\pi}(\boldsymbol{s})=E\left[\sum_{t=0}^{\infty} \gamma^{t} R\left(\boldsymbol{s}_{t}\right) \mid \boldsymbol{s}_{0}=\boldsymbol{s}, \pi\right],
$$

As it is well-known the value function must obey the Bellman's equation:

$$
V^{\pi}(s)=E_{\pi}\left[R\left(s_{t}\right)+\gamma V^{\pi}\left(s_{t+1}\right) \mid s_{t}=s\right] .
$$

which expresses a relationship between the values of successive states in the same episode. Similarly, the state-action value function ( $Q$-function) $Q(s, a)$ denotes the expected cumulative reward as received by taking action $a$ in state $s$ following the policy $\pi$.

$$
Q^{\pi}(\boldsymbol{s}, a)=E_{\pi}\left[\sum_{t=0}^{\infty} \gamma^{t} R\left(\boldsymbol{s}_{t}\right) \mid \boldsymbol{s}_{0}=\boldsymbol{s}, a_{0}=a\right] .
$$

In this study we will be focused on $Q$ functions dealing with state-action pairs $(s, a)$.

The objective of RL problems is to estimate an optimal policy $\pi^{*}$ by choosing actions that yields the optimal action-state value function $Q^{*}$ :

$$
\pi^{*}(s)=\arg \max _{a} Q^{*}(s, a) .
$$

A common choice for representing the value function is through a linear function approximation using a set of $k$ basis functions $\left\{\phi_{j}(s, a)\right\}_{j}$ :

$$
Q(s, a ; \boldsymbol{w})=\phi(s, a)^{\top} \boldsymbol{w}=\sum_{j=1}^{k} \phi_{j}(s, a) w_{j},
$$

where $\boldsymbol{w}=\left(w_{1}, \ldots, w_{k}\right)$ is a vector of weights which are unknown and must be estimated so as to minimize the approximation error. The selection of the basis functions is very important and must be chosen to encode properties of the state and action relevant to the proper determination of the $Q$ values. As we will se later, our method provides an adaptive incremental procedure for discovering basis functions through online clustering.

One of the most popular TD algorithms used in on-policy RL is the SARSA [1] that is based on bootstrapping technique. Assuming that an action $a_{t}$ is taken and the agent moves from belief state $s_{t}$ to a new state $\boldsymbol{s}_{t+1}$ while receiving a reward $r_{t}$, a new action $a_{t+1}$ is chosen according to the current policy. Then, the predicted $Q$ value of this new state is used to calculate an improved estimate for the $Q$ value of the previous state:

$$
\delta_{t}=r_{t}+\gamma Q\left(s_{t+1}, a_{t+1}\right)-Q\left(s_{t}, a_{t}\right)=r_{t}+\gamma\left(\phi\left(s_{t+1}, a_{t+1}\right)-\phi\left(s_{t}, a_{t}\right)\right)^{\top} \boldsymbol{w}_{t},
$$


which is the scalar one-step temporal-difference (TD) error. This is used next for adjusting the weights of the policy by performing a stochastic gradient descent scheme:

$$
\boldsymbol{w}_{t+1}=\boldsymbol{w}_{t}+\alpha_{t} \delta_{t} \nabla_{\boldsymbol{w}} Q\left(\boldsymbol{s}_{t}, a_{t}\right),
$$

where $\alpha_{t}$ is the learning rate which set to some small value (e.g. 0.05) and can be decreased over time. Note that sometimes it is useful to use the version of SARSA with the eligibility traces, $\operatorname{SARSA}(\lambda)$, that allows the update rule to propagate rewards backward over the current trajectory of states during the episode. This is done by modifying the above equation (Eq. 7) as $\boldsymbol{w}_{t+1}=\boldsymbol{w}_{t}+\alpha_{t} \delta_{t} e_{t}$, where $e_{t}=\gamma \lambda e_{t-1}+\nabla_{\boldsymbol{w}} Q\left(\boldsymbol{s}_{t}, a_{t}\right)$ is the vector of eligibility traces with $\lambda$ being a parameter in $[0,1]$. This is the version we have adopted in our approach.

\section{The proposed method}

The proposed methodology is based on a policy evaluation scheme that incrementally builds a dictionary of basis functions for modeling the value functions. This is accomplished by using an online clustering scheme that decomposes appropriately an efficient kernel space of the inputs so as to achieve optimal exploration of the value function. To what follows and for simplicity we will assume that the input samples that are generated by the agent are state-action pairs, denoted as $x_{i}=\left(s_{i}, a_{i}\right)$. We will also consider that the action space is discrete of size $M$.

Assuming a given data set of $N$ samples $\left\{x_{1}, x_{2}, \ldots, x_{N}\right\}$ the task of clustering aims at partitioning the input set into $k$ disjoint clusters, $c_{1}, c_{2}, \ldots, c_{k}$ containing samples with common properties. The kernel $k$-means $[15,16]$, is an extension of the standard $k$-means algorithm that is based on transforming data to a feature space through appropriate kernel functions and minimizing the clustering error in this space. In particular, the objective function is given by

$$
J_{k}=\sum_{i}^{N} \min _{j=1}^{k}\left\{-K\left(x_{i}, m_{j}\right)\right\}
$$

where $m_{j}$ are some representatives for each cluster. In our study we have considered that every cluster $c_{j}$ is characterized by the following features:

- $\boldsymbol{\mu}_{j}:$ its centroid in state space $\mathcal{S}$,

- $\Sigma_{j}$ : diagonal covariance matrix over the state space,

- $\boldsymbol{p}_{j}=\left(p_{j 1}, \ldots, p_{j M}\right)$ : the density function over the $M$ discrete actions, giving the probabilities of each action $\left(\sum_{m=1}^{M} p_{j m}=1\right)$.

These features constitute the representative vector $m_{j}$ for every cluster.

The kernel function $K\left(x_{i}, m_{j}\right)$ for an arbitrary sample, $x_{i}=\left(\boldsymbol{s}_{i}, a_{i}\right)$, generated by the agent with the $c_{j}$ cluster that has a representative vector $m_{j}=$ $\left(\boldsymbol{\mu}_{j}, \Sigma_{j}, p_{j}\right)$ is derived as a product of two kernels, one for each space:

$$
K\left(x_{i}, m_{j}\right)=K_{s}\left(\boldsymbol{s}_{i}, \boldsymbol{\mu}_{j}, \Sigma_{j}\right) K_{a}\left(a_{i}, \boldsymbol{p}_{j}\right),
$$


For the state space we have used Gaussian-type kernel:

$$
K_{s}\left(\boldsymbol{s}_{i}, \boldsymbol{\mu}_{j}, \Sigma_{j}\right)=\exp \left(\left(\boldsymbol{s}_{i}-\boldsymbol{\mu}_{j}\right)^{\top} \Sigma_{j}^{-1}\left(\boldsymbol{s}_{i}-\boldsymbol{\mu}_{j}\right)\right),
$$

while for the action space the kernel function is derived from the probability for this action of the cluster action distribution, i.e.

$$
K_{a}\left(a_{i}, \boldsymbol{p}_{j}\right)=p_{j, a_{i}} .
$$

Note that in fact the above kernel function in the action space is the cosine similarity between the probability vector for actions and an indicator vector of the input action $a_{i}$ with zeros in all positions except for the position of $a_{i}$ that has one.

In our case the samples are non-stationary and are generated sequentially (i.e. time-varying). Online clustering provides a framework for constructing recursive learning rules taking into account model evolutions over time. The proposed online kernel-based clustering method is performed iteratively as follows: For a random taken data point $x_{i}=\left(s_{i}, a_{i}\right)$, the method first selects the winning cluster $j^{*}$ according to the current kernel values, i.e.

$$
j^{*}=\arg \max _{j=1}^{k} K\left(x_{i}, m_{j}\right) .
$$

If the maximum kernel value is less than a predefined threshold value $K_{\min }$, then a new cluster is created $k=k+1$ by initializing it properly. This is done by setting the state $s_{i}$ as the cluster state centroid, $\boldsymbol{\mu}_{k}=s_{i}$, while for the action density probability $\boldsymbol{p}_{k}$ we give a large value for the action probability of action $a_{i}$ (e.g. $p_{k, a_{i}}=0.8$ ) and normalize the others so as to hold the constraint $\sum_{m} p_{k m}=1$.

The next step is the adaptation phase where the prototype $m_{j^{*}}$ of the winning cluster must be adjusted. This is accomplished by using the next update rules:

$$
\begin{gathered}
\boldsymbol{\mu}_{j^{*}}^{(n e w)}=\boldsymbol{\mu}_{j^{*}}+\eta K\left(x_{i}, m_{j}\right)\left(\boldsymbol{s}_{i}-\boldsymbol{\mu}_{j^{*}}\right), \\
\Sigma_{j^{*}}^{(n e w)}=\Sigma_{j^{*}}+\eta K\left(x_{i}, m_{j}\right) \operatorname{diag}\left(\left(\boldsymbol{s}_{i}-\boldsymbol{\mu}_{j^{*}}^{(n e w)}\right)\left(\boldsymbol{s}_{i}-\boldsymbol{\mu}_{j^{*}}^{(n e w)}\right)^{\top}\right), \\
n_{j m}^{n e w}= \begin{cases}n_{j m}+1, & \text { if } m=a_{i} \\
n_{j m}, & \text { otherwise }\end{cases}
\end{gathered}
$$

where the term $\eta$ is the learning rate taking a small value (e.g. 0.05) and which again can be reduced over time. It must be noted that the density of actions $p_{j m}$ is guided by the frequency distribution $n_{j m}$ and thus it is more convenient to keep record of frequencies. Then, the probabilities $p_{j m}$ are calculated by the relative frequencies. From the above rule, it is easily to show that the probability of action $a_{i}$ will be increased by $\left(\nu_{j}-n_{j m}\right) /\left(\nu_{j}\left(\nu_{j}+1\right)\right.$, while the probability of the other $M-1$ actions will be decreased by $-n_{j m} /\left(\nu_{j}\left(\nu_{j}+1\right)\right.$, where $\nu_{j}=\sum_{m=1}^{M} n_{j m}$ is the total frequency of cluster $c_{j}$. 
The above procedure is repeated until convergence, or the number of episodes reaches a prespecified value. The method starts with a single cluster $k=1$, where it is initialized as described previously by the first data point seen by the agent $x_{1}=\left(s_{1}, a_{1}\right)$. At every time step the policy evaluation stage uses the $k$ basis functions as (currently) taken by the clustering procedure. Therefore, the online clustering approach provides not only the shape, but also the proper number of basis functions for estimating the value function. At a second level, the linear weights are re-estimated following the temporal difference (TD) learning process, as described previously.

Algorithm 1 summarizes the overall scheme of the proposed methodology.

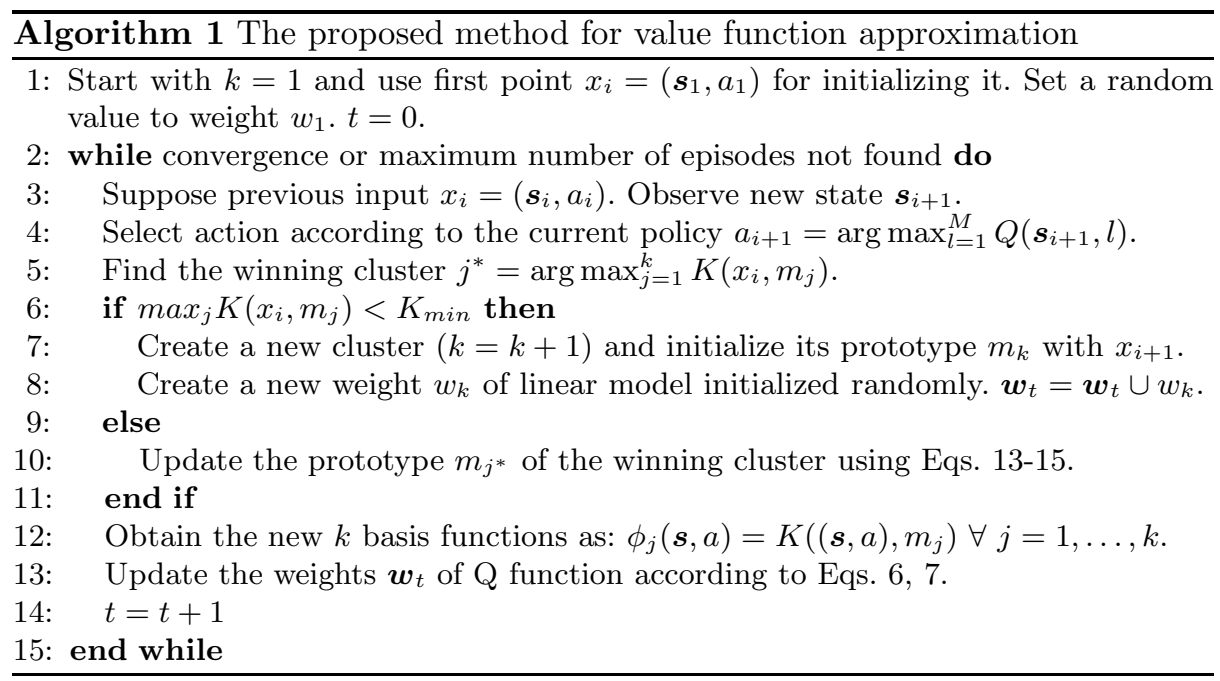

\section{Experimental results}

A number of experiments have been conducted using three well-known continuous benchmarks in order to assess the performance of the proposed methodology. These environments can be found on the RL-Glue software which are freely available at http://glue.rl-community.org/. Comparison has been made with a recent method presented in [10] that uses fixed Fourier basis functions of order 3 (denoted as ' $\mathrm{O}(3)$ Fourier') ${ }^{1}$. This methd In all experiments we have set the discount factor $\gamma$ equal to 1 , the parameter $\lambda$ equal to 0.9 , and the threshold for adding a new cluster as $K_{\text {min }}=0.5$.

The first benchmark is the famous cart pole where the objective is to keep the pole balanced and the cart within its limits by applying a fixed magnitude force either to the left, or to the right (two actions). There are four continuous

\footnotetext{
${ }^{1}$ open source code for this method can be found in the RL-Glue library
} 
variables: the horizontal position and the velocity of the cart, as well as the angle and the angular velocity of the pole, while the reward received is +1 .

The second environment is the mountain car, where the objective is to drive an under-powered car up a steep mountain road from a valley to the right tophill using three actions. The state consists of two continuous variables: the position and the current velocity of the car, while at every step a negative reward $r=-1$ is received.

In the last domain the agent controls a simulated acrobot attached by the hands to a fixed location. The goal is to apply torque to the hips of the robot and swing the feet above a pre-specified threshold. There are four continuous variables: the angle and the angular velocity of the two joints. The agent can select between three actions: positive torque, negative torque and zero torque on the second joint. A negative reward $(r=-1)$ is received at each time step except for the case where the goal is reached $(r=0)$. An episode is terminated only when the goal is reached.
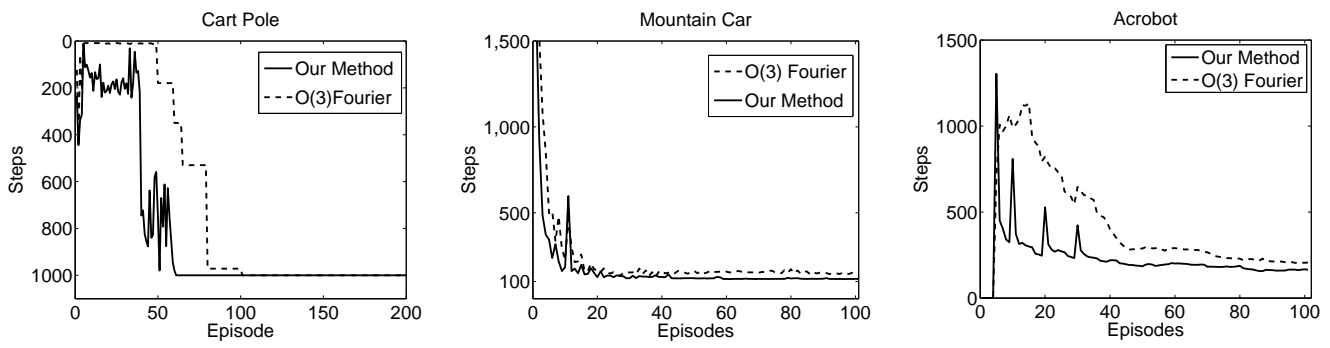

Fig. 1. Comparative results in three simulated environments.

The depicted results on these three benchmarks are illustrated in Fig. 1, where each curve gives the number of steps that the agent makes per episode. Note these are the mean curves obtained by 10 runs per problem. As it is obvious our method has the tendency to converge to the optimum solution faster than the 'O(3)Fourier' method that employs (256) fixed Fourier basis function. It is interesting to note that in the case of the 'mountain car' and the 'acrobot' environments the proposed managed to discover additionally better policy.

\section{Conclusions and future directions}

In this study we have presented a novel framework for learning representation of reinforcement learning agents and control in Markov decision processes. An online kernel-based clustering approach is used as a mechanism for creating and adjusting clusters over the input state-action pairs generated by the agent. At each step, the current cluster parameters are used for building an efficient kernel space that provides with appropriate basis functions to the temporal-difference 
learning framework. Thus, the linear weights used for value function approximation are sequentially adjusted in a more optimal way. The initial results of our method obtained from the comparative study are very promising and promote directions for further research: Since the proposed scheme of constructing basis functions is general, it allows the possibility to study its impact to other temporal difference algorithms for learning the weights of the value function, such as the Least-Squares Temporal Difference (LSTD) or Gaussian Process Temporal Difference (GPTD). Also, alternative schemes of online clustering can be examined, as well as to make an extensive comparison with other value function approximation approaches.

\section{References}

1. R.S. Sutton and A.G. Barto. Reinforcement Learning: An Introduction. MIT Press Cambridge, USA, 1998.

2. L.P. Kaelbling, M.L. Littman, and A.W. Moore. Reinforcement learning: A survey. Journal of Artificial Inteligence Research, 4:237-285, 1996.

3. R. Sutton. Learning to predict by the method of temporal differences. Machine Learning, 3(1):9-44, 1988.

4. J. A. Boyan. Technical update: Least-squares temporal difference learning. $M a-$ chine Learning, pages 233-246, 2002.

5. M. G. Lagoudakis and R. Parr. Least-squares policy iteration. Journal of Machine Learning Research, 4:1107-1149, 2003.

6. X. Xu, D. Hu, and X. Lu. Kernel-based least squares policy iteration for reinforcement learning. IEEE Transactions on Neural Networks, 18(4):973-992, 2007.

7. C.E. Rasmussen and M. Kuss. Gaussian processes in reinforcement learning. In Advances in Neural Information Processing Systems 16, pages 751-759, 2004.

8. Y. Engel, S. Mannor, and R. Meir. Reinforcement learning with gaussian process. In International Conference on Machine Learning, pages 201-208, 2005.

9. A. M. Farahmand, M. Ghavamzadeh, C. Szepesvári, and S. Mannor. Regularized policy iteration. In NIPS, pages 441-448, 2008.

10. G.D. Konidaris, S. Osentoski, and P.S. Thomas. Value function approximation in reinforcement learning using the fourier basis. In AAAI Conf. on Artificial Intelligence, pages 380-385, 2011.

11. S. Mahadevan. Samuel meets amarel: Automating value function approximation using global state space analysis. In $A A A I, 2005$.

12. S. Mahadevan and M. Maggioni. Proto-value Functions: A Laplacian Framework for Learning Representation and Control in Markov Decision Processes. Journal of Machine Learning Research, 8:2169-2231, 2007.

13. I. Menache, S. Mannor, and N. Shimkin. Basis Function Adaptation in Temporal Difference Reinforcement Learning. Annals of Operations Research, 134:215-238, 2005.

14. M. Petrik. An analysis of laplacian methods for value function approximation in mdps. In International Joint Conference on Artificial Intelligence, pages 25742579, 2007.

15. B. Scholkopf, A. J. Smola, and K.-R. Muller. Nonlinear component analysis as a kernel eigenvalue problem. Neural Computation, 10(5):1299-1319, 1998.

16. G. Tzortzis and A. Likas. The Global Kernel k-Means Clustering Algorithm. IEEE Trans on Neural Networks, 20(7):1181-1194, 2009. 